\title{
Spectral Collocation Technique for Absorbing Boundary Conditions with Increasingly High Order Approximation
}

\author{
Zhenli $\mathrm{Xu}$ and Houde Han \\ Department of Mathematics, University of Science and Technology of China, \\ Hefei, Anhui 230026, China \\ xuzl@ustc.edu
}

\begin{abstract}
An efficient treatment is developed for the Schrödinger equation with a class of local absorbing boundary conditions, which are obtained by high order Padé expansions. These boundary conditions are significant in the simulation of open quantum devices. Based on the finite difference approximation in the interior domain, we construct a spectral collocation layer on the cell near the artificial boundary, in which the wave function is approximated by the Chebyshev polynomials. The numerical examples are given by using this strategy with increasingly high order of accuracy up to the ninth order.
\end{abstract}

Keywords: Absorbing boundary conditions, Schrödinger equation, semiconductor devices, Chebyshev spectral collocation, difference schemes.

\section{Introduction}

For numerical simulations of quantum mechanical models for semiconductor devices, it is important and challenging to design an effective open boundary condition which absorbs outgoing waves [1. In this paper, we consider high-order local absorbing boundary conditions (ABCs) for the Schrödinger equation

$$
i \hbar \psi_{t}(x, t)=-\frac{1}{2} \hbar^{2} \Delta \psi(x, t)+V(x, t) \psi(x, t), \quad x \in \mathbb{R}^{d},
$$

which models quantum waveguides and resonant tunneling structures. Here we assume that the initial data $\psi(x, 0)=f(x)$ is compactly support in a closed region $\Omega$, and the given potential $V$ is assumed to be a constant $V_{0}$ outside $\Omega$ so that there is no incoming wave on the truncated boundaries $\partial \Omega$.

One way is to construct the transparent boundary condition (TBC) by approximating the exact solution of the exterior problem restricted to the finite computational domain $\Omega$. There are many papers $1,2,3,4,6,7,8$ developing TBCs and studying their difference approximations and stability. However, the obtained TBC through this way is nonlocal in $t$, thus requiring all the history data in memory. Moreover, the computational effort of an ad-hoc discretization is unacceptable high. 
To attain a method with low computational cost, another way is to construct ABCs 910111213 by approximating the nonlocal operator in TBC with polynomials. This class of boundary conditions is local in time, and easy to implement. In addition, they can be applied to nonlinear problems such as optical fiber communications [14 through a local time-splitting approach [15]. An efficient implementation of $\mathrm{ABCs}$ with high-order rational polynomial approximations is urgently required for the accurate solution of the Schrödinger equation (for a recent review paper, see Hagstrom [16]). However, it is also very difficult due to the high-order derivatives of the ABCs and their weak illposedness 12 . For this, we construct a spectral collocation layer on the cell near the boundary. The Chebyshev spectral method, which is very efficient for high-order equations [1718, can be practically implemented in the layer, together with finite difference discretizations in interior domain.

The organization of this paper is as follows. In $\S 2$, high-order local ABCs are formulated. In $\S 3$, numerical issues are discussed. In $\S 4$, several numerical examples are given to illustrate the performance of the method. $\S 5$ gives some concluding remarks.

\section{Construction of Absorbing Boundary Conditions}

Consider the one-dimensional Schrödinger equation

$$
i \psi_{t}(x, t)=-\psi_{x x}(x, t)+V(x, t) \psi(x, t),
$$

which models the transient behavior of electrons in a quantum waveguide. Here the coefficients are absorbed into the derivatives for simplicity. We truncate the unbounded domain to a finite one $[-L, L]$ so that the initial value $f(x)=0$ and the potential $V(x, t)=V_{0}$ for $|x| \geq L$.

We concentrate on the right boundary. Similar discussion can be performed on the left boundary. General wave packets propagating to the right at $x=L$ are represented by

$$
\psi(x, t)=\int_{V_{0}}^{+\infty} e^{i\left(\sqrt{\omega-V_{0}} x-\omega t\right)} \hat{\psi}(x, \omega) d \omega,
$$

where $\hat{\psi}$, satisfying $\hat{\psi}(L, \omega)=0$ for $\omega<V_{0}$, denotes the Fourier transform in $t$ under the dual relation $\omega \leftrightarrow i \partial_{t}$ between the frequency domain and the time domain. From Eq. (3), the TBC [1] in transformed space

$$
i \hat{\psi}_{x}+\sqrt{\omega-V_{0}} \hat{\psi}=0
$$

which annihilates all the outgoing waves, is given by

$$
\psi_{x}(L, t)=-\sqrt{\frac{1}{\pi}} e^{-i\left(\frac{\pi}{4}+V_{0} t\right)} \frac{d}{d t} \int_{0}^{t} \psi(L, \tau) e^{i V_{0} \tau} \frac{d \tau}{\sqrt{t-\tau}} .
$$

It is a nonlocal boundary condition. In order to get local boundary conditions which allow a minor reflection, we approximate the square root $\sqrt{\omega-V_{0}}$ by using 
polynomials or rational polynomials. Note that $k=\sqrt{\omega-V_{0}}$ is positive. The zero- and first-order Taylor approximations centered $\omega_{0}$ with $k_{0}=\sqrt{\omega_{0}-V_{0}}>0$ to the square root give

$$
\begin{aligned}
& \sqrt{\omega-V_{0}}=k_{0}+O\left(\omega-\omega_{0}\right), \\
& \sqrt{\omega-V_{0}}=k_{0}+\frac{\omega-V_{0}}{2 k_{0}}
\end{aligned}
$$

where the wavenumber parameter $k_{0}$ can be adaptively picked through a windowed Fourier transform [19. These approximations at once lead to the firstand second order ABCs

$$
\begin{aligned}
& B_{1} \psi=i \psi_{x}+k_{0} \psi=0, \\
& B_{2} \psi=i \psi_{t}+i 2 k_{0} \psi_{x}+\left(2 k_{0}^{2}-V_{0}\right) \psi=0 .
\end{aligned}
$$

Higher-order Taylor approximations usually lead to ill-posed problems as shown for the hyperbolic wave equation in Engquist and Majda 20] and for the convection-diffusion equation in Halpern [21, in which the authors showed that a hierarchy of Padé approximations shall lead to well-posed problems. For the Schrödinger equation (2), the $(n, n)$-Padé approximations to $\sqrt{\omega-V_{0}}$ can be deduced through a recursive relation, as

$$
\sqrt{\omega-V_{0}}=k_{0} P_{n} / Q_{n}+O\left(\omega-\omega_{0}\right)^{2 n+1},
$$

with $P_{0}=Q_{0}=1$ and

$$
\begin{aligned}
& P_{n}=\left(\omega-V_{0}+k_{0}^{2}\right) P_{n-1}+2\left(\omega-V_{0}\right) Q_{n-1}, \\
& Q_{n}=\left(\omega-V_{0}+k_{0}^{2}\right) Q_{n-1}+2 k_{0}^{2} P_{n-1} .
\end{aligned}
$$

And for $(n+1, n)$-Padé approximations, the recursive relation is

$$
\sqrt{\omega-V_{0}}=k_{0} P_{n} / Q_{n}+O\left(\omega-\omega_{0}\right)^{2 n+2},
$$

with $P_{0}=\omega-V_{0}+k_{0}^{2}, Q_{0}=2 k_{0}^{2}$ and

$$
\begin{aligned}
& P_{n}=\left(\omega-V_{0}+k_{0}^{2}\right) P_{n-1}+2\left(\omega-V_{0}\right) Q_{n-1}, \\
& Q_{n}=\left(\omega-V_{0}+k_{0}^{2}\right) Q_{n-1}+2 k_{0}^{2} P_{n-1} .
\end{aligned}
$$

We let $\left\{\tilde{P}_{n}, \tilde{Q}_{n}\right\}$ represent the dual operators in physical space of $\left\{P_{n}, Q_{n}\right\}$ in frequency domain. Then after transforming Eqs. (6) and (7) back to the physical space by using the dual relation and using Eq. (4), we obtain high order approximations to the TBC: for $(2 n+1)$-st order ABC by the $(n, n)$-Padé approximation,

$$
B_{2 n+1} \psi=\left(i \tilde{Q}_{n} \partial_{x}+k_{0} \tilde{P}_{n}\right) \psi=0,
$$

with $\tilde{P}_{0}=\tilde{Q}_{0}=1$, and

$$
\begin{aligned}
& \tilde{P}_{n}=\left(i \partial_{t}-V_{0}+k_{0}^{2}\right) \tilde{P}_{n-1}+2\left(i \partial_{t}-V_{0}\right) \tilde{Q}_{n-1}, \\
& \tilde{Q}_{n}=\left(i \partial_{t}-V_{0}+k_{0}^{2}\right) \tilde{Q}_{n-1}+2 k_{0}^{2} \tilde{P}_{n-1} ;
\end{aligned}
$$


and, for $(2 n+2)$-nd order ABC by the $(n+1, n)$-Padé approximation,

$$
B_{2 n+2} \psi=\left(i \tilde{Q}_{n} \partial_{x}+k_{0} \tilde{P}_{n}\right) \psi=0
$$

with $\tilde{P}_{0}=i \partial_{t}-V_{0}+k_{0}^{2}, \tilde{Q}_{0}=2 k_{0}^{2}$, and the same recursive formulae as (9) and (10). For the $(n, n+1)$-Padé approximation, we remark that the obtained ABCs are ill-posed, as is discussed in Alonso-Mallo and Reguera [12].

\section{Numerical Approximation}

\subsection{Discretization of Interior Domain}

Denote the approximation of $\psi$ on the grid point $\left(x_{j}, t^{n}\right)$ by $\psi_{j}^{n}$ for $0 \leq j \leq J$, with $x_{j}=x_{0}+j \Delta x$ and $t^{n}=n \Delta t$. In the interior domain, the Schrödinger equation is approximated by the Crank-Nicholson scheme

$$
i \frac{\psi_{j}^{n+1}-\psi_{j}^{n}}{\Delta t}=\left(-D^{+} D^{-}+V_{j}^{n+\frac{1}{2}}\right) \frac{\psi_{j}^{n+1}+\psi_{j}^{n}}{2}, \quad 1 \leq j \leq J-1,
$$

where $D^{+}$and $D^{-}$represent the forward and backward differences, respectively.

\subsection{Order Reduction for Time Derivatives of Boundary Conditions}

In order to numerically discrete the boundary conditions with high order derivatives, it is necessary to reduce the time derivatives to first order. Thus, a twolayer scheme can be easily performed. For this, we use the original equation $i \psi_{t}=-\psi_{x x}+V_{0} \psi$ to substitute $\partial_{t}$ with terms $\psi_{x x}$ and $\psi$. For example, for the $(n, n)$-Padé approximations, the boundary condition $B_{3} \psi=0$ is of the first order with respect to $t$ when $n=1$. And when $n>1$, we can write the operators $\tilde{P}_{n}$ and $\tilde{Q}_{n}$ as

$$
\tilde{P}_{n}=\left(-\partial_{x x}+k_{0}^{2}\right) \tilde{P}_{n-1}-2 \partial_{x x} \tilde{Q}_{n-1}, \quad \tilde{Q}_{n}=\left(-\partial_{x x}+k_{0}^{2}\right) \tilde{Q}_{n-1}+2 k_{0}^{2} \tilde{P}_{n-1} .
$$

The boundary condition $B_{2 n+1} \psi=0$ then keeps first order with respect to $t$. Therefore, the midpoint rule can be easily achieved as a time discretization method.

\subsection{Approximation of Spectral Collocation Layers}

We now concentrate on the discretizations of boundary conditions, in which we shall use a technique of the spectral collocation method (for the theory and application of spectral methods, see monographs [22 23] for details). The collocation points are distributed on the cell near the artificial boundary; i.e., on $\left[x_{0}, x_{1}\right]$ at the left boundary and $\left[x_{J-1}, x_{J}\right]$ at the right. We consider the right boundary again. The approximation of difference scheme (12) at $x_{J-1}$ can be regarded as a boundary condition of the right collocation domain, and thus the collocation domain is well-posed together with the ABC (8) or (11) at $x_{J}$. 
To introduce the spectral collocation method, we transform the domain $\left[x_{J-1}, x_{J}\right]$ into $[-1,1]$ by using scaling

$$
\xi=s\left[x-\left(x_{J}+x_{J-1}\right) / 2\right] \text {, with } s=2 /\left(x_{J}-x_{J-1}\right) .
$$

Eq. (2) near the boundary is then written as

$$
i \tilde{\psi}_{t}(\xi, t)=-s^{2} \tilde{\psi}_{\xi \xi}(\xi, t)+V_{0} \tilde{\psi}(\xi, t),
$$

where $\tilde{\psi}(\xi, t)=\psi(x, t)$. Similarly, the derivatives in the recursive formulae of ABCs can be scaled. Let $T_{m}(\xi)=\cos (m \arccos (\xi))$ for $0 \leq m \leq M$ be the Chebyshev polynomials, and $\xi_{m}=\cos (m \pi / M)$ be the Chebyshev-Gauss-Labatto collocation points. The Chebyshev interpolating polynomial in space with $M$ collocation points reads

$$
\left(\mathcal{P}_{M} \tilde{\psi}\right)(\xi, t)=\sum_{m=0}^{M} \tilde{\psi}_{m}(t) \phi_{m}(\xi),
$$

where $\tilde{\psi}_{m}(t)$ represents the point value of $\tilde{\psi}(\xi, t)$ at $\xi_{m}$, and the basis function is

$$
\phi_{m}(\xi)=\frac{2}{\alpha_{m} M} \sum_{l=0}^{M} \frac{1}{\alpha_{l}} T_{l}\left(\xi_{m}\right) T_{l}(\xi),
$$

with $\alpha_{m}=1$, except for $\alpha_{0}=\alpha_{M}=2$. If we approximate the $p$-th spatial derivatives of $\tilde{\psi}(\xi, t)$ at the collocation points, $\xi_{m}$, we have

$$
\tilde{\boldsymbol{\psi}}^{(p)}=D^{(p)} \tilde{\boldsymbol{\psi}}=D^{p} \tilde{\boldsymbol{\psi}}
$$

where $\tilde{\boldsymbol{\psi}}$ denotes the vector $\tilde{\boldsymbol{\psi}}=\left[\tilde{\psi}\left(\xi_{0}\right), \tilde{\psi}\left(\xi_{1}\right), \cdots, \tilde{\psi}\left(\xi_{M}\right)\right]^{T}$. The corresponding differentiation matrix $D$ has the entries

$$
\begin{aligned}
& D_{m l}=\frac{\alpha_{m}}{\alpha_{l}} \frac{(-1)^{m+l}}{\xi_{m}-\xi_{l}}, \quad m \neq l, \\
& D_{m m}=-\frac{\xi_{m}}{2\left(1-\xi_{m}^{2}\right)}, \quad 0<m<M \\
& D_{00}=-D_{M M}=\frac{2 M^{2}+1}{6} .
\end{aligned}
$$

Using the above approximations in space and the midpoint rule in time, the equation in the collocation layer can be expressed as a system of algebraic equations which is connected with scheme (12) in the interior domain at $x=x_{J-1}$. The formed algebraic system is a modified triangular linear matrix which contains two blocks in the upper left and lower right corners.

\section{Numerical Examples}

In this section, we shall give some numerical examples for the Schrödinger equation (2) in homogeneous media; i.e., we set $V \equiv 0$. 
Example 1. In the first example, we are going to consider the evolvement of the right travelling Gaussian beam $\psi(x, 0)=e^{-x^{2}} e^{i q x}$ with $q=5$ in a truncated region $[-10,10]$. Similar tests can be found in 12 and in 24, which simulated optical beam propagation in the Fresnel approximation. By taking the parameter $k_{0}=q$ and $2 q$ respectively in the calculations, we compute the solution up to $T=10$, and calculate the reflection ratio $r=\sum_{j=0}^{J}\left|\psi_{j}^{n}\right|^{2} / \sum_{j=0}^{J}\left|\psi_{j}^{0}\right|^{2}$ at time $t^{n}=T$. In Tables 1 and 2, we show the results of the reflection ratios for different boundary conditions with increasing high order approximation for $(n, n)$-Padé and $(n+1, n)$-Padé polynomials, respectively. We see that a higher order boundary condition has usually a better effect of absorbing outgoing waves. The instability of the numerical solution also increases with the order of the ABCs. For the eighth and ninth order ABCs, blowups appear; and the behavior is worse, the smaller grid size is. However, these results are reasonable due to the weak ill-posedness of the high order ABCs which induce ill-conditioned matrices. Therefore, a better solver is desired to the linear systems.

Table 1. Reflection ratios with $\Delta x=\Delta t$ for the difference scheme, and $M$ collocation points for the spectral collocation layer

\begin{tabular}{|c|c|c|c|c|}
\hline & & \multicolumn{2}{|r|}{$k_{0}=5$} & $k_{0}=10$ \\
\hline$\Delta x$ & $M$ & $\overline{\text { Padé- }(1,1)}$ & $\overline{(2,2)}$ & $\overline{(2,2)}$ \\
\hline 0.1 & 13 & $7.58 \mathrm{e}-5$ & $5.69 \mathrm{e}-55.49 \mathrm{e}-5 \quad 5.38 \mathrm{e}-5$ & $1.87 \mathrm{e}-4$ 7.63e-5 $6.33 \mathrm{e}-5 \quad 5.78 \mathrm{e}-5$ \\
\hline & 17 & $7.58 \mathrm{e}-5$ & 5.69e-5 5.49e-5 Blowup & $1.87 \mathrm{e}-4 \quad 7.63 \mathrm{e}-5 \quad 6.33 \mathrm{e}-5 \quad 7.17 \mathrm{e}-5$ \\
\hline 0.05 & 13 & $1.59 \mathrm{e}-5$ & $5.60 \mathrm{e}-63.89 \mathrm{e}-6$ Blowup & $9.19 \mathrm{e}-5$ 2.50e-5 1.17e-5 Blowl \\
\hline & 17 & $1.59 \mathrm{e}-5$ & 5.60e-6 3.89e-6 Blowup & $9.19 \mathrm{e}-5$ 2.50e-5 1.16e-5 Blowu \\
\hline
\end{tabular}

Table 2. $(n+1, n)$-Padé approximations with the same settings as Table 1

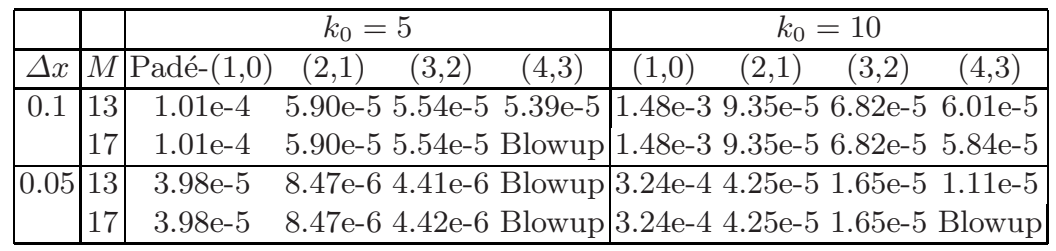

Example 2. The second example is for the initial data

$$
\psi(x, 0)=e^{-x^{2}} \int_{0}^{5}\left(e^{i q x}+e^{-i q x}\right) / 2 d q=e^{-x^{2}} \sin (5 x) / x
$$

in a truncated region $[-10,10]$, which is composed of waves with different group velocities. We compute the solution up to $T=2$. The parameter $k_{0}$ in the boundary conditions is now a function of time; we take a linear function $k_{0}(t)=(11-t) / 2$, because at time $t$ the components with $\pm k_{0}(t)$ wavenumbers arrive at the boundaries. In the calculations, we take $M=13, \Delta x=0.05$ and $\Delta t=0.005$. The 
numerical solution with the same mesh sizes in a large domain $[-40,40]$ is taken to be a reference "exact" solution, since the analytic solution is unknown. The numerical solutions for ABCs up to seventh order are illustrated in Fig. 1. It is a good example to show why high-order ABCs are required. We see from the curves that lower-order ABCs reflect many outgoing waves which pollute the solution in the computational domain, and that higher-order ABCs demonstrate better results.
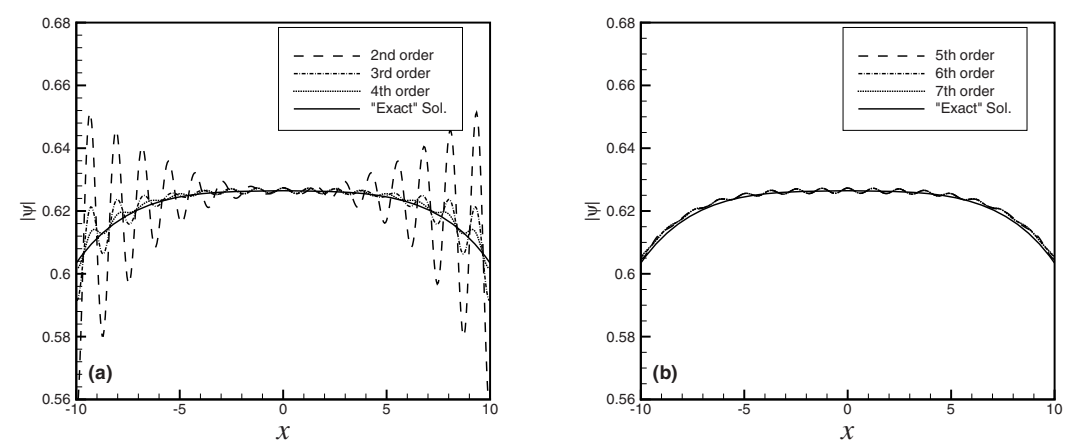

Fig. 1. Numerical results of wave packets $|\psi|$ at $t=2$ for different ABCs

\section{Concluding Remarks}

High-order absorbing boundary conditions (ABCs) for the linear Schrödinger equation are implemented through using a spectral collocation technique. $\mathrm{Nu}-$ merical results illustrate the attractive performance of higher-order cases. However, the instability appears with the increase of the order, which motivates further investigation on the improvement of the approach. The method is outlined for one-dimensional problem but the extensions to multidimensional cases of the technique are straightforward in Cartesian grids. Even so, a detailed report for multidimensional problems is also necessary, which is under consideration as a possible part of the future research.

Acknowledgments. This work is supported by the National Natural Science Foundation of China (Grant Nos. 10471073 and 40674037).

\section{References}

1. Arnold, A.: Numerically absorbing boundary conditions for quantum evolution equations. VSLI Design 6 (1998) 313-319

2. Schmidt, F., Yevick, D.: Discrete transparent boundary conditions for Schrödingertype equations. J. Comput. Phys. 134 (1997) 96-107

3. Ehrhardt, M.: Discrete transparent boundary conditions for general Schrödingertype equations. VLSI Design 9(4) (1999) 325-338 
4. Arnold, A., Ehrhardt, M., Sofronov, I.: Discrete transparent boundary conditions for the Schrödinger equation: Fast calculation, approximation, and stability. Commun. Math. Sci. 1 (2003) 501-556

5. Antoine, X., Besse, C.: Unconditionally stable discretization schemes of nonreflecting boundary conditions for the one-dimensional Schrödinger equation. J. Comput. Phys. 188 (2003) 157-175

6. Han, H., Huang, Z.: Exact artificial boundary conditions for Schrödinger equation in $R^{2}$. Commun. Math. Sci. 2 (2004) 79-94

7. Han, H., Jin, J., Wu, X.: A finite-difference method for the one-dimensional timedependent Schrödinger equation on unbounded domain. Comput. Math. Appl. 50 (2005) 1345-1362

8. Sun, Z.Z., Wu, X.: The stability and convergence of a difference scheme for the Schrödinger equation on an infinite domain by using artificial boundary conditions. J. Comput. Phys. 214 (2006) 209-223

9. Shibata, T.: Absorbing boundary conditions for the finite-difference time-domain calculation of the one dimensional Schrödinger equation. Phys. Rev. B 43 (1991) 6760

10. Kuska, J.P.: Absorbing boundary conditions for the Schrödinger equation on finite intervals. Phys. Rev. B 46 (1992) 5000

11. Fevens, T., Jiang, H.: Absorbing boundary conditions for the Schrödinger equation. SIAM J. Sci. Comput. 21 (1999) 255-282

12. Alonso-Mallo, I., Reguera, N.: Weak ill-posedness of spatial discretizations of absorbing boundary conditions for Schrödinger-type equations. SIAM J. Numer. Anal. 40 (2002) 134-158

13. Szeftel, J.: Design of absorbing boundary conditions for Schrödinger equations in $R^{d}$. SIAM J. Numer. Anal. 42 (2004) 1527-1551

14. Agrawal, G.: Nonlinear fiber optics, 3rd Ed. Academic Press, San Diego (2001)

15. Xu, Z., Han, H.: Absorbing boundary conditions for nonlinear Schrödinger equations. Phys. Rev. E 74 (2006) 037704

16. Hagstrom, T.: New results on absorbing layers and radiation boundary conditions. Lect. Notes Comput. Sci. Eng. 31 (2003) 1-42

17. Elbarbary, E.M.E., El-Sayed, S.M.: Higher order pseudospectral differentiation matrices. Appl. Numer. Math. 55 (2005) 425-238

18. Mai-Duy, N.: An effective spectral collocation method for the direct solution of high-order ODEs. Commun. Numer. Meth. Eng. 22 (2006) 627-642

19. Xu, Z., Han, H., Wu, X.: Adaptive absorbing boundary conditions for Schrödingertype equations: application to nonlinear and multi-dimensional problems. J. Comput. Phys., to appear (2007) arXiv: math.NA/0610642.

20. Engquist, B., Majda, A.: Radiation boundary conditions for acoustic and elastic wave calculations. Commun. Pure Appl. Math. 32 (1979) 313-357

21. Halpern, L.: Artificial boundary conditions for the linear advection diffusion equation. Math. Comput. 46(174) (1986) 425-438

22. Trefethen, L.N.: Spectral Methods in MATLAB. SIAM: Philadelphia, PA (2000)

23. Shen, J., Tang, T.: Spectral and High-Order Methods with Applications. Science Press, Beijing (2006)

24. Yevick, D., Yu, J.: Optimal absorbing boundary conditions. J. Opt. Soc. Am. A 12 (1995) 107-110 